\title{
Neuroprotective effect of calcitriol on ischemic/reperfusion injury through the NR3A/CREB pathways in the rat hippocampus
}

\author{
JUN FU*, RUI XUE, JUNFENG GU, YUN XIAO, HEYING ZHONG, XUELIAN PAN and RAN RAN* \\ Department of Anesthesiology, Renmin Hospital, Hubei University of Medicine, Shiyan, Hubei 442000, P.R. China
}

Received March 25, 2013; Accepted September 23, 2013

DOI: $10.3892 / \mathrm{mmr} .2013 .1734$

\begin{abstract}
Calcitriol has been demonstrated to provide neuroprotection against ischemia/reperfusion (I/R) injury. However, the exact mechanism of this protection remains unknown. In the present study, the neuroprotective effect of calcitriol was investigated in rats exposed to cerebral I/R injury induced by middle cerebral artery occlusion (MCAO). In addition, the involvement of NR3A, extracellular signal-regulated kinase 1/2 (ERK1/2), and phosphorylated cAMP/Ca ${ }^{2+}$-response element binding protein (p-CREB) in this protective action was determined in the hippocampal neurons. Western blot analysis was conducted to analyze the protein levels of NR3A, mitogen-activated protein kinase kinase (MEK) and p-CREB. The immunoreactivity of $\mathrm{p}-\mathrm{CREB}$ and NR3A were measured by quantum dot-based immunofluorescence analysis. Results showed that MCAO rats exhibited large cortical infarct volumes. By contrast, intraperitoneal administration of calcitriol significantly reduced infarct volumes seven days following reperfusion, and these results were accompanied by elevated NR3A and p-CREB activity in the hippocampal neurons. The inhibition of MEK by the addition of PD98059 led to attenuation of the neuroprotective effects of calcitriol and a correlated decrease in CREB activity. The results also demonstrated that calcitriol protected the brain from I/R injury through the NR3A-MEK/ERK-CREB pathway.
\end{abstract}

\section{Introduction}

Stroke is a leading global cause of morbidity and mortality (1). Ischemic stroke occurs when the blood supply to the brain is obstructed, and the majority of ischemic strokes result from acute thrombosis. Currently, tissue plasminogen activator (tPA) is the only approved agent by the Food and Drug

Correspondence to: Dr Ran Ran, Department of Anesthesiology, Renmin Hospital, Hubei University of Medicine, 39 Chaoyang Avenue, Shiyan, Hubei 442000, P.R. China

E-mail: ranran1146@163.com

*Contributed equally

Key words: cerebral ischemia, cAMP/ $\mathrm{Ca}^{2+}$-response element binding protein, neuroprotection, NR3A, rat
Administration for ischemic stroke treatment; however, tPA must be administered within $4.5 \mathrm{~h}$ of stroke onset for it to exert therapeutic effects (2). Thus, tPA has limited applicability and is currently used in $<5 \%$ of stroke victims (3). Therefore, studies are required to identify therapies with an increased efficacy and extended treatment window for stroke patients.

The N-methyl-D-aspartate (NMDA) receptor (NMDAR) complex is a tetrameric or pentameric structure composed of at least two NR1 subunits and two or three subunits from the NR2 family (NR2A-D) (4,5). An additional NMDA receptor subunit, NR3A, has been identified in mammalian brains $(6,7)$. However, unlike the conventional NR1/NR2 receptors, those containing NR3 subunits exhibit decreased single-channel conductance, insensitivity to magnesium blockade and reduced calcium $\left(\mathrm{Ca}^{2+}\right)$ permeability (7-10). NR3 subunits act in a novel, dominant-negative manner to suppress NMDAR activity $(6,11)$.

Calcitriol is the biologically active metabolite of vitamin D and the predominant $\mathrm{Ca}^{2+}$-regulatory steroid hormone in peripheral tissues $(12,13)$. Previous studies have demonstrated that the chronic peripheral treatment of rats with calcitriol retarded the age-related decrease in neuronal density observed in the rodent hippocampus (14) and protected against damage in a rodent model of stroke (15). In addition, a previous study also demonstrated that calcitriol exhibits a direct consistent neuroprotective action against excitotoxic insults (16).

The aim of this study was to determine whether calcitriol protected the brain from ischemic injury through a signaling mechanism involving elevated levels of NR3A and $\mathrm{Ca}^{2+}$-response element binding protein ( $\mathrm{p}-\mathrm{CREB}$ ), and to determine whether mitogen-activated protein kinase kinase (MEK)/extracellular signal-regulated kinase (ERK) is involved in the regulatory mechanism of NR3A-mediated p-CREB expression.

\section{Materials and methods}

Animals and treatment. Healthy male Sprague-Dawley rats (weight, 200-250 g) were purchased from Hunan Weasleyg Scene of Experimental Animals Co., Ltd. (Changsha, China). Experimental protools were approved by the Ethics Committee of Tongji Medical College, Huazhong University of Science and Technology (Wuhan, China), and conformed to internationally accepted ethical standards (Guide For the Care and Use of Laboratory Animals; NIH Publication 85-23, 
revised 1985). The rats were allowed access to food and water ad libitum. Rats were randomly divided into four groups $(n=12)$ : Sham-operated rats (group S); rats with middle cerebral artery occlusion (MCAO) (group I); rats with MCAO followed by calcitriol treatment (group $\mathrm{C}$ ); and rats with MCAO followed by calcitriol plus PD98059 treatment (group P) (Fig. 1).

Calcitriol (Cayman Chemical Company, Ann Arbor, MI, USA) was dissolved in ethanol and diluted with $0.9 \% \mathrm{NaCl}$ solution immediately prior to intraperitoneal (i.p.) administration. The drug was applied either acutely (a single dose of $2 \mu \mathrm{g} / \mathrm{kg}$, immediately following ischemia) and subchronically ( $2 \mu \mathrm{g} / \mathrm{kg}$ on six consecutive days). Control animals (groups I and $\mathrm{S}$ ) received $0.9 \% \mathrm{NaCl}$ supplemented with the required volume of ethanol. On day six, the final dose was administered $1 \mathrm{~h}$ prior to surgery. An MEK inhibitor, PD98059 (dissolved in $1 \%$ dimethylsulfoxide; $0.75 \mathrm{mg} / \mathrm{rat}$ ), was administered alone or in combination with calcitriol.

Focal cerebral ischemia. Stroke was induced using the intraluminal filament MCAO model (17). Throughout the surgical procedure, rectal temperature was monitored and maintained at $37^{\circ} \mathrm{C}$ using a circulating heating pad. Briefly, the animals were anesthetized with $10 \%$ chloral hydrate $(400 \mathrm{mg} / \mathrm{kg}$, i.p.), and the right common carotid artery (CCA) and its proximal branches were isolated. The CCA and external carotid artery were ligated, and the internal carotid artery (ICA) was temporarily occluded using a metal microvessel clip. A nylon monofilament (Beijing Sunbio Biotech Co., Ltd., Beijing, China) with a rounded tip was inserted and advanced through the CCA and ICA until resistance was felt. The filament was left in place for $2 \mathrm{~h}$ and then withdrawn. Rats in the sham-operated group were subjected to the same surgical procedure, however, they did not undergo MCAO. All animals were placed in a warm environment until they had fully recovered from the anesthesia.

Measurement of the infarct volume. For 2,3,5-triphenyltetrazolium chloride (TTC) staining, brain tissues were sectioned into 2-mm thick coronal slices seven days following reperfusion. These tissues were stained for $20 \mathrm{~min}$ in a $2 \%$ TTC solution (Sigma-Aldrich, St. Louis, MO, USA) and fixed in $4 \%$ paraformaldehyde. The stained tissues were photographed by a digital camera (COOLPIX P500; Nikon, Tokyo, Japan) and measured for ischemic lesions by Image $\mathrm{J}$ software (National Institutes of Health, Bethesda, MD, USA). The ischemic lesion percentage of each slice was calculated by the ratio of the infarction area to the whole slice area.

Western blot analysis. Subsequent to seven days of reperfusion, the rats were euthanized by decapitation and the hippocampal tissues were harvested. Total protein extraction was performed using the total protein extraction kit (Nanjing Keygen Biotech Co., Ltd., Nanjing, China). Total protein extracts were prepared for protein determination and analyzed by western blot analysis for NR3A, phosphorylated MEK (p-MEK) and MEK. Nuclear protein extraction was performed according to the manufacturer's instructions (Fermentas International, Glen Burnie, MD, USA). Nuclear protein extracts were prepared to determine the expression of p-CREB. Protein concentration was analyzed by a bicin- choninic acid assay kit (Nanjing Keygen Biotech Co., Ltd.). Equal quantities of protein were loaded and separated by sodium dodecyl sulfate-polyacrylamide gel electrophoresis and transferred onto a polyvinylidene difluoride membrane. The membrane was blocked and incubated overnight at $4^{\circ} \mathrm{C}$ with the following antibodies: Anti-NR3A $(1: 1,000$; Millipore, Billerica, MA, USA), anti-phospho-MEK, anti-MEK, anti-p-CREB (dilution, 1:1,000; Cell Signaling Technology Inc., Beverly, MA, USA), anti-Lamin B1 (dilution, 1:500; Bioworld Merchandising, Inc., Minneapolis, MN, USA) and anti-GAPDH (dilution, 1:1,000; Proteintech Group, Inc, Chicago, IL, USA). Following three washes with Tris-buffered saline and Tween 20 for $15 \mathrm{~min}$, the membrane was incubated with the appropriate horseradish peroxidaseconjugated secondary antibodies (dilution, 1:5,000) for $1 \mathrm{~h}$ at room temperature. Labeled proteins were detected with the ChemiDoc XRS chemiluminescence imaging system (Bio-Rad, Hercules, CA, USA). Protein bands were quantified by Image $\mathrm{Lab}^{\mathrm{TM}}$ image acquisition and analysis software (Bio-Rad). The experiments were repeated in triplicate.

Quantum dot-based immunofluorescence. Subsequent to seven days of reperfusion, the animals were anesthetized with chloral hydrate $(400 \mathrm{mg} / \mathrm{kg}$, i.p.) and perfused transcardially with $0.9 \%$ sodium chloride at $4{ }^{\circ} \mathrm{C}$, followed by $4 \%$ paraformaldehyde in $0.1 \mathrm{M}$ phosphate buffer ( $\mathrm{pH} 7.4$ ). The brains were then rapidly removed, blocked and embedded in paraffin. Paraffin-embedded brains were cut into 4 - $\mu \mathrm{m}$-thick sections according to standard procedures. The paraffin sections $(n=3)$ were incubated overnight with antibodies against NR3A (dilution, 1:100; Millipore) at $4^{\circ} \mathrm{C}$, following blocking with bovine serum albumin (BSA). The samples were then incubated with a biotinylated secondary antibody at $37^{\circ} \mathrm{C}$ for $30 \mathrm{~min}$. Following blocking with BSA, the paraffin sections were incubated with streptavidin-conjugated QDs605 (dilution, 1:100; Wuhan Jiayuan Quantum Dot Technological Development, Co, Ltd., Wuhan, Hubei, China). NR3A-positive cells were measured at x200 magnification per visual field in the cortex; three visual fields per section and three brain sections per rat were analyzed. Fluorescent signals were detected with a fluorescence microscope (BX51; Olympus, Tokyo, Japan) and signal intensities were collected for statistical analysis. Images were captured with a Doppler imaging system (CRi Nuance Fx; Caliper Life Sciences, Hopkinton, MA, USA).

Statistical analysis. All values are presented as the mean \pm standard error of the mean. One-way analysis of variance followed by a post hoc Newman-Keuls test was performed for statistical comparison of several groups. The unpaired t-test was used for the comparison of two groups. $\mathrm{P}<0.05$ was considered to indicate a statistically significant difference. GraphPad Prism for Windows (version 5; GraphPad Software Inc., San Diego, CA, USA) was used for all statistical analyses.

\section{Results}

Effect of calcitriol on infarct area and volume following focal cerebral ischemia. Seven days following ischemia/reperfusion (I/R), rats developed infarcts affecting the cortex and striatum (Fig. 2). The calcitriol treatment group had a significantly 


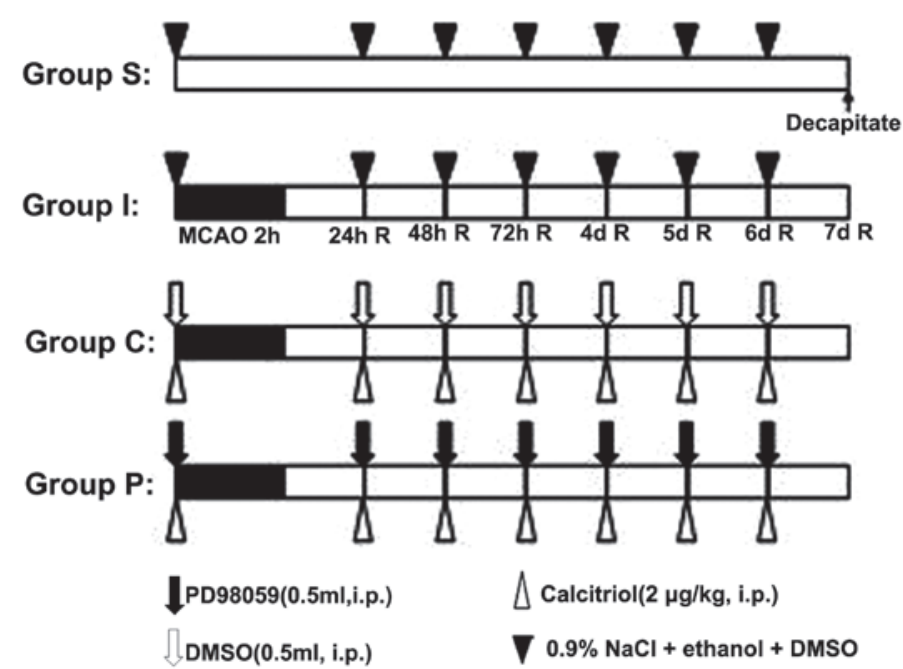

Figure 1. Experimental protocol used to determine the effect of calcitriol following ischemia and reperfusion. The rats were randomly divided into four groups (n=3): Group S, sham-operated; group I, MCAO; group C, MCAO followed by calcitriol treatment; Group P, MCAO followed by calcitriol plus PD98059 (MEK inhibitor) treatment. MCAO, middle cerebral artery occlusion; MEK, mitogen-activated protein kinase kinase.

A
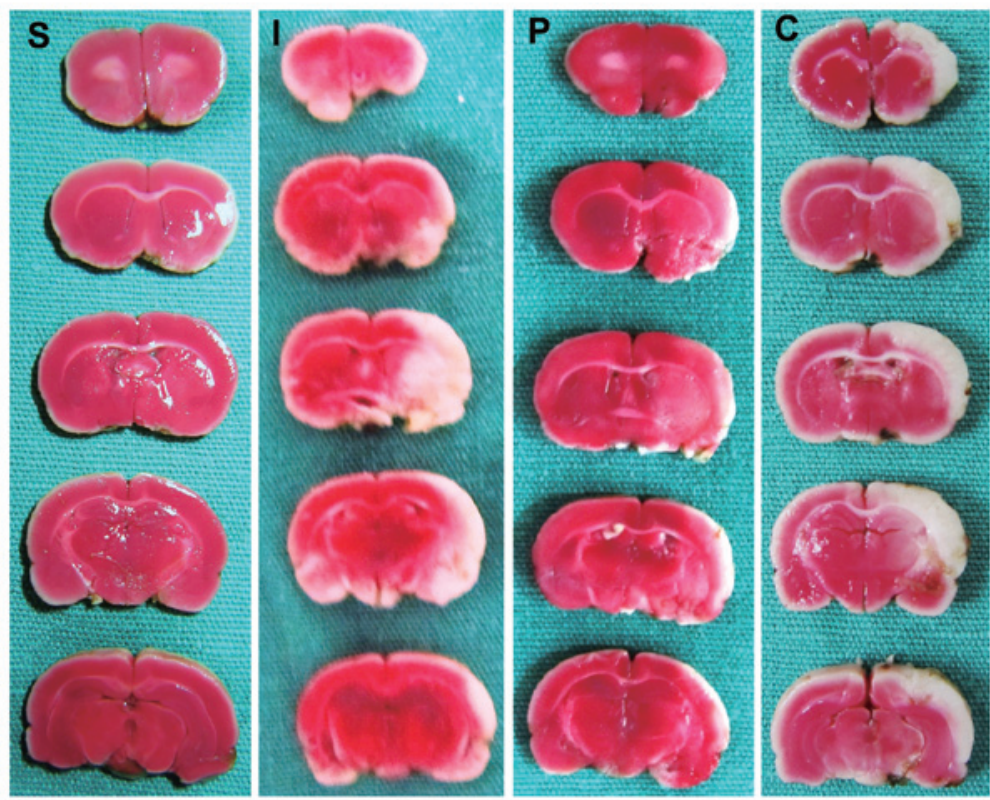

B

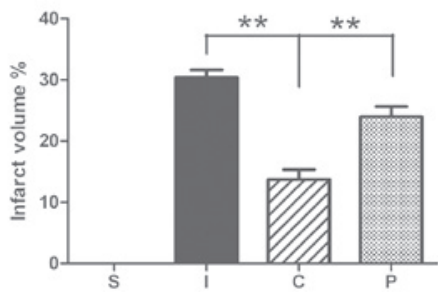

Figure 2. Effect of calcitriol on the infarct volumes and neurological scores following seven days of transient MCAO. (A) Representative 2,3,5-triphenyltetrazolium chloride staining of the cerebral infarct in the rat brain $(n=6)$. (B) Statistical analysis of the percentage of infarct volume was determined for each study group. Calcitriol-treated rats significantly reduced infarct volumes compared with the MCAO group. With application of PD98059, the infarct volume was significantly increased compared with the calcitriol-treated group. Bars represent the mean $\pm \mathrm{SEM}$. ** $\mathrm{P}<0.01$. Group $\mathrm{S}$, sham-operated; group I, MCAO; group C, MCAO followed by calcitriol treatment; Group P, MCAO followed by calcitriol plus PD98059 (MEK inhibitor) treatment. MCAO, middle cerebral artery occlusion; MEK, mitogen-activated protein kinase kinase.

smaller infarct area and volume of total hemisphere infarction seven days following MCAO compared with those of the control (P<0.01; Fig. 2).
Calcitriol reduces the degradation of NR3A in I/R injury. To determine whether calcitriol exerted a neuroprotective effect through the regulation of the NR3A level, the NR3A 
A

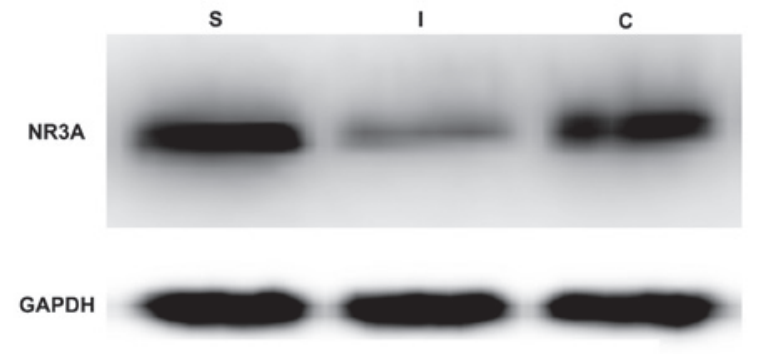

B

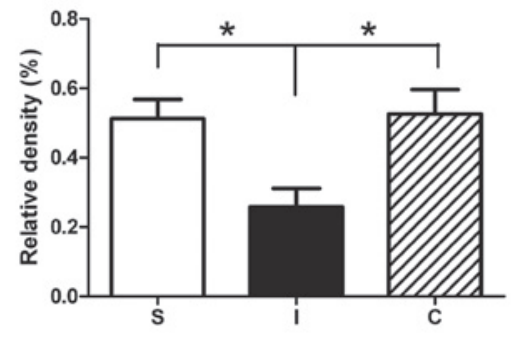

Figure 3. Effect of calcitriol on the levels of NR3A seven days following reperfusion. (A) Western blot analysis of NR3A. Total protein extracts were prepared from the ipsilateral hippocampus. GAPDH was used as an internal reference. (B) Densitometric analysis of the protein levels of NR3A in the ipsilateral cortex $(\mathrm{n}=3)$. Values are presented as the mean $\pm \mathrm{SEM}$. " $\mathrm{P}<0.05$. Group $\mathrm{S}$, sham-operated; group I, MCAO; group $\mathrm{C}, \mathrm{MCAO}$ followed by calcitriol treatment. MCAO, middle cerebral artery occlusion; GAPDH, glyceraldehyde 3-phosphate dehydrogenase.

A DG
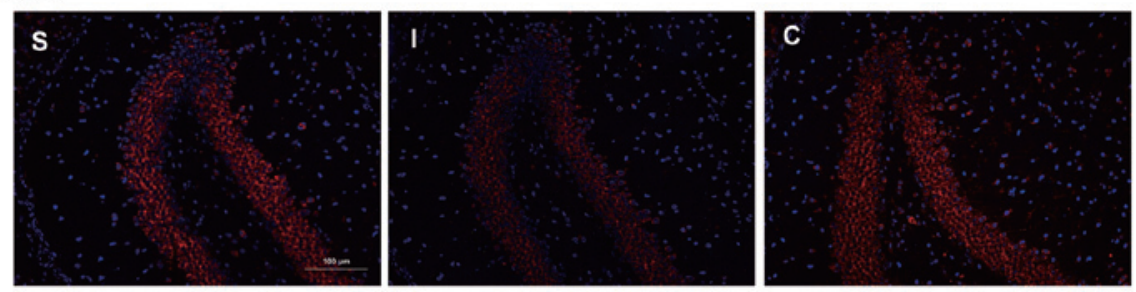

\section{B CA3}
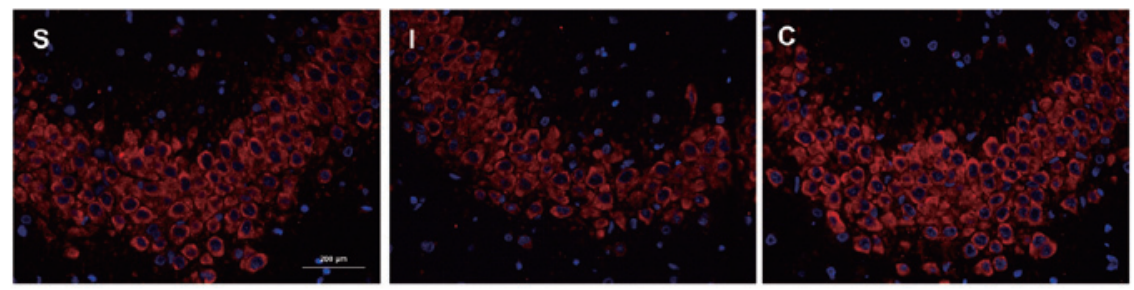

Figure 4. Effect of calcitriol treatment on the immunoreactivity of NR3A seven days following reperfusion. (A) NR3A immunoreactivity in the hippocampal dentate gyrus area $(\mathrm{n}=3)$. Staining was present within the cell membrane. Scale bar, $100 \mu \mathrm{m}$. (B) NR3A immunoreactivity in the hippocampal CA3 area (n=3). Scale bar, $200 \mu \mathrm{m}$. Group S, sham-operated; group I, MCAO; group C, MCAO followed by calcitriol treatment ischemia combined with calcitriol treatment. MCAO, middle cerebral artery occlusion.

levels in the rat hippocampus were investigated by western blot analysis (Fig. 3). In the hippocampal tissues collected seven days following ischemia, the NR3A expression level was significantly reduced in group I compared with that of the sham-operated animals (Fig. 3). Treatment with calcitriol significantly restored the level of NR3A to that observed in the uninjured rats.

Immunofluorescence results showed a cytomembrane staining pattern of NR3A protein in neurons of the hippocampal dentate gyrus and CA3 areas, and these findings were corroborated by the results of the western blot analysis (Fig. 4).
PD98059 specifically inhibits MEK phosphorylation. To determine the effectiveness and specificity of the MEK inhibitor PD98059, p-MEK levels in the rat hippocampus were determined by western blot analysis (Fig. 5). Subsequent to the application of PD98059, the protein levels of p-MEK in the rat hippocampus were significantly decreased seven days following reperfusion compared with those of the calcitriol-treated group $(\mathrm{P}<0.01 ;$ Fig. 5).

Calcitriol maintains the level of $p$-CREB via the NR3A-MEK/ERK pathway. In the hippocampal tissues collected seven days following ischemia, the p-CREB expres- 
A

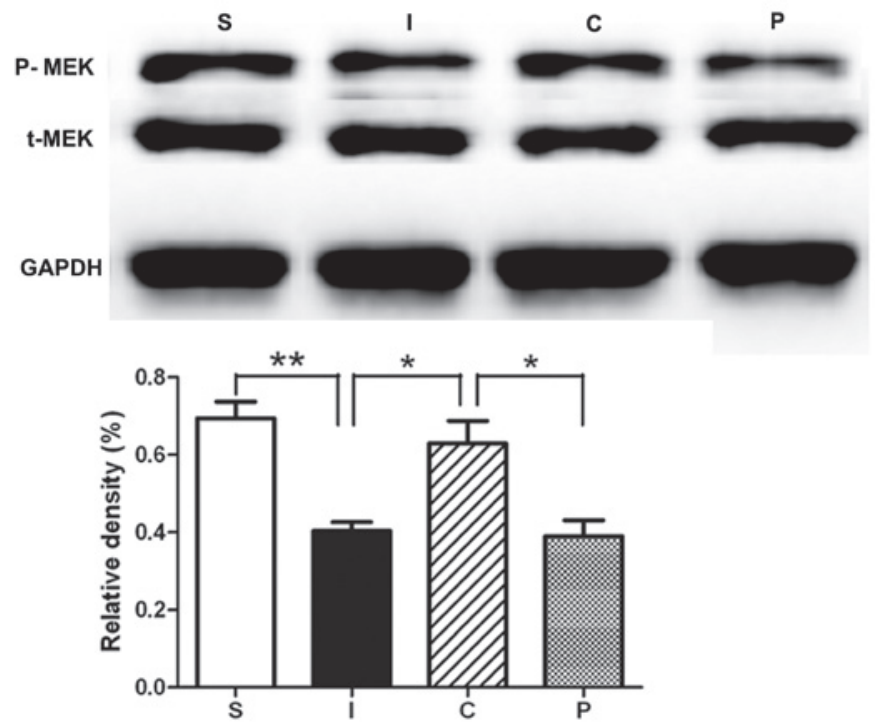

Figure 5. Inhibitory effect of PD9805 on MEK assessed with the protein levels of MEK seven days following reperfusion. Samples were prepared from the ipsilateral hippocampus. Western blot analysis was performed using specific antibodies against the indicated proteins. Blots were reprobed for total-MEK and GAPDH to normalize each lane for protein content. (A) Western blot analysis of phospho-MEK in the ipsilateral hippocampus at the indicated times or sham surgery. (B) Densitometric analysis of the protein levels of phospho-MEK in the ipsilateral hippocampus ( $\mathrm{n}=3$ rats for each condition). Values are presented as the mean \pm SEM. ${ }^{*} \mathrm{P}<0.05$ and ${ }^{* *} \mathrm{P}<0.01$. Group S, sham-operated; group I, MCAO; group C, MCAO followed by calcitriol treatment; Group P, MCAO followed by calcitriol plus PD98059 (MEK inhibitor) treatment. MEK, mitogen-activated protein kinase kinase; GADPH, glyceraldehyde 3-phosphate dehydrogenase; MCAO, middle cerebral artery occlusion.

A

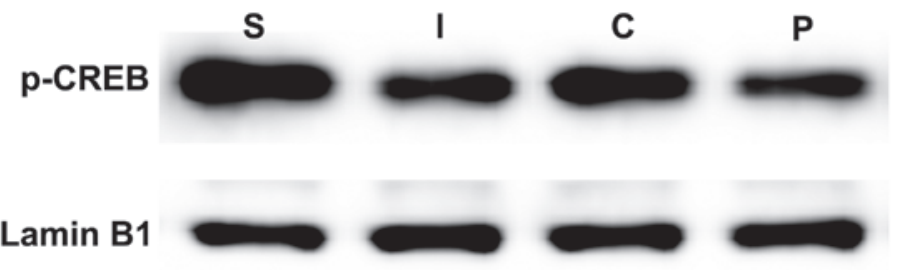

$\mathbf{B}$

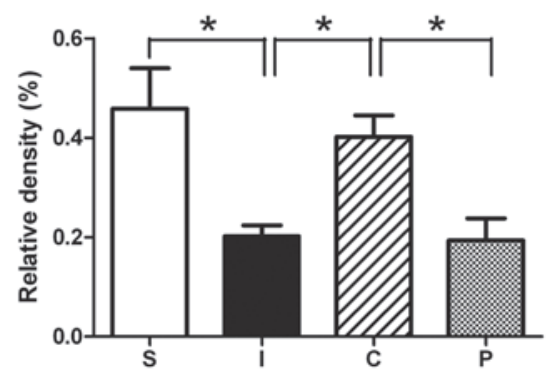

Figure 6. Effect of calcitriol treatment on the protein levels of p-CREB seven days following reperfusion. (A) Western blot analysis of p-CREB. Nuclear protein extracts were prepared from the ipsilateral hippocampus. Lamin B1 was used as an internal reference. A 43-kDa band corresponding to p-CREB protein was clearly detected. (B) Densitometric analysis of the protein levels of p-CREB in the ipsilateral hippocampus $(n=3)$. Values are the mean \pm SEM. "P $<0.05$. Group S, sham-operated; group I, MCAO; group C, MCAO followed by calcitriol treatment; Group P, MCAO followed by calcitriol plus PD98059 (MEK inhibitor) treatment. p-CREB, phosphorylated cAMP/ $/ \mathrm{Ca}^{2+}$-response element binding protein; $\mathrm{MCAO}$, middle cerebral artery occlusion; MEK, mitogen-activated protein kinase kinase.

sion levels in the rat hippocampus were significantly reduced compared with those of the sham-operated animals. The treatment with calcitriol significantly restored the levels of $\mathrm{p}-\mathrm{CREB}$ to those of the uninjured rats. This upregulation was prevented by the inhibition of the ERK (PD98059) pathway (Fig. 6).

\section{Discussion}

The present results demonstrated that the NR3A subunit was effective in protecting the brain from ischemic injury. In this study, treatment with calcitriol for seven days significantly decreased the infarct volumes, and was correlated with elevated NR3A and p-CREB activities, following cerebral I/R injury. This neuroprotective effect was attenuated by cotreatment with PD98059, an MEK (the upstream kinase of ERK) inhibitor. Therefore, the results clearly demonstrated that calcitriol exerted neuroprotective effects against ischemic injury through the NR3A-MEK/ERK-CREB pathways.

In stroke, excessive extracellular glutamate overstimulates glutamate receptors, initiating excessive calcium entry mainly 
through the NMDARs, which is the predominant contributory factor to neuronal excitotoxicity injury during the process of ischemic stroke $(18,19)$. NMDARs are molecularly organized as heteromeric complexes incorporating different subunits of three subtypes: NR1, NR2 and NR3, the latter of which has two subunits (NR3a and NR3b) (20). In vitro and in vivo studies have suggested that NMDAR antagonists are effective in ischemic neuronal death, and pharmacological agents that block glutamate release or glutamate-mediated postsynaptic excitability are able to reduce neural degeneration in rat stroke models $(21,22)$. However, studies concerning the discovery of neuroprotective agents in the last few decades focused on NMDAR antagonists, which although promising in preclinical studies, failed during clinical trials $(23,24)$. Among numerous possible reasons for this failure, it is suggested that the NR2A-containing NMDARs mediate neuronal survival while the NR2B-containing NMDARs are coupled to neuronal apoptosis. Blockade of the NR2A-containing NMDARs does not confer neuroprotection, and, by contrast leads to the exacerbation of neuronal death. However, blocking NR2B-mediated cell death was effective in reducing infarct volume only when the receptor antagonist was administered prior to the onset of stroke and not $4.5 \mathrm{~h}$ subsequent to stroke $(25,26)$. Therefore, the common conception concerning treatment of ischemic brain damage with NMDAR antagonists may have to be reconsidered. Conventional NMDARs are composed of NR1 and NR2 subunits, while the incorporation of NR3A gives the NMDAR unconventional properties, such as low $\mathrm{Ca}^{2+}$ permeability and decreased sensitivity to $\mathrm{Mg}^{2+}$ blocking (27). NR3A subunits modulate the susceptibility of oligodendroglial lineage NMDARs to glycine or D-serine activation $(28,29)$. In situ hybridization and immunohistochemistry analyses have demonstrated that the NR3A subunit is widely distributed in the rat brain with predominant expression of the novel NR3B subunit by motor neurons (30). The co-expression of NR3A and NR3B subunits prevents $\mathrm{Ca}^{2+}$ mobilization into the mitochondria following the activation of NMDAR channels composed of NR1/NR2A and NR1/NR2B subunits on the cell surface, in association with the rescue from cell death (31). Cultured neurons expressing transgenic (TG) NR3A exhibited greater resistance to NMDA-mediated neurotoxicity than wild type (WT) neurons. Similarly, in vivo, adult NR3A TG mice subjected to focal cerebral ischemia exhibited less damage than WT mice (32). A previous study has demonstrated that calcitriol provides neuroprotection against I/R injury (15). In addition, calcitriol has a direct and highly consistent neuroprotective action against excitotoxic insults (16). Therefore, the results suggest that calcitriol may protect the brain from ischemic injury through a signaling mechanism involving elevated levels of NR3A. In the present study, the variation of NR3A in the MCAO group suggested that brain ischemic injury induced downregulation of NR3A in the hippocampal CA1 region; however, calcitriol treatment reversed this tendency and significantly increased the NR3A levels.

The nuclear transcription factor CREB, active form p-CREB, exhibits numerous functions. The phosphorylation of serine-133 in CREB allows it to interact with the co-activator, CREB-binding protein/p300, and is required for its activation. A previous study demonstrated that $\mathrm{p}$-CREB stimulated neurogenesis and prevented infarct expansion in the penumbra region of cerebral ischemia (33). The CREB activation was a critical event in neuroprotection against ischemic injury $(34,35)$ suggesting that the NR3A subunit may rescue neurons from glutamate excitotoxicity mediated by NMDAR through activation of CREB. In the present study, calcitriol treatment markedly reduced the brain infarct area and enhanced the expression levels of NR3A and p-CREB. In addition, PD98059 was used to investigate the pathway by which calcitriol protected rats from cerebral ischemia. It was demonstrated that when PD98059 was co-administered with calcitriol, the p-CREB protein levels were significantly decreased seven days following reperfusion compared with the levels measured in calcitriol-treated rats. These results demonstrated that the activation of CREB through the MEK/ERK pathway is a pivotal downstream effector for the protective effect of the NR3A subunit in neurons.

In conclusion, MCAO rats receiving calcitriol treatment exhibited a markedly reduced brain infarct area and enhanced expression levels of NR3A and p-CREB. Furthermore, $\mathrm{MEK} / \mathrm{ERK}$ is involved in the regulatory mechanism of NR3A-mediated p-CREB expression. The results may provide insights into the pleiotropic role of calcitriol and the functional modulation of NR3A, and provide a basis for the protective effect of calcitriol on brain ischemia.

\section{References}

1. Feigin VL: Stroke epidemiology in the developing world. Lancet 365: 2160-2161, 2005.

2. Fisher M, Feuerstein G, Howells DW, et al: Update of the stroke therapy academic industry roundtable preclinical recommendations. Stroke 40: 2244-2250, 2009.

3. Grotta JC, Burgin WS, El-Mitwalli A, et al: Intravenous tissue-type plasminogen activator therapy for ischemic stroke: Houston experience 1996 to 2000. Arch Neurol 58: 2009-2013, 2001.

4. McBain CJ and Mayer ML: N-methyl-D-aspartic acid receptor structure and function. Physiol Rev 74: 723-760, 1994.

5. Dingledine R, Borges K, Bowie D and Traynelis SF: The glutamate receptor ion channels. Pharmacol Rev 51: 7-61, 1999.

6. Sucher NJ, Akbarian S, Chi CL, et al. Developmental and regional expression pattern of a novel NMDA receptor-like subunit (NMDAR-L) in the rodent brain. J Neurosci 15: 6509-6520, 1995.

7. Chatterton JE, Awobuluyi M, Premkumar LS, et al: Excitatory glycine receptors containing the NR3 family of NMDA receptor subunits. Nature 415: 793-798, 2002.

8. Das S, Sasaki YF, Rothe T, et al: Increased NMDA current and spine density in mice lacking the NMDA receptor subunit NR3A. Nature 393: 377-381, 1998.

9. Perez-Otano I, Schulteis CT, Contractor A, et al: Assembly with the NR1 subunit is required for surface expression of NR3A-containing NMDA receptors. J Neurosci 21: 1228-1237, 2001.

10. Sasaki YF, Rothe T, Premkumar LS, et al: Characterization and comparison of the NR3A subunit of the NMDA receptor in recombinant systems and primary cortical neurons. J Neurophysiol 87: 2052-2063, 2002.

11. Ciabarra AM, Sullivan JM, Gahn LG, Pecht G, Heinemann S and Sevarino KA: Cloning and characterization of chi-1: a developmentally regulated member of a novel class of the ionotropic glutamate receptor family. J Neurosci 15: 6498-6508, 1995.

12. DeLuca HF and Zierold C: Mechanisms and functions of vitamin D. Nutr Rev 56: S4-S10, (discussion S54-S75), 1998.

13. Brown AJ, Dusso A and Slatopolsky E: Vitamin D. Am J Physiol 277: F157-F175, 1999.

14. Landfield PW and Cadwallader-Neal L: Long-term treatment with calcitriol $(1,25(\mathrm{OH}) 2$ vit D3) retards a biomarker of hippocampal aging in rats. Neurobiol Aging 19: 469-477, 1998.

15. Wang Y, Chiang YH, Su TP, et al: Vitamin D(3) attenuates cortical infarction induced by middle cerebral arterial ligation in rats. Neuropharmacology 39: 873-880, 2000. 
16. Brewer LD, Thibault V, Chen KC, Langub MC, Landfield PW and Porter NM: Vitamin D hormone confers neuroprotection in parallel with downregulation of L-type calcium channel expression in hippocampal neurons. J Neurosci 21: 98-108, 2001

17. Longa EZ, Weinstein PR, Carlson S and Cummins R: Reversible middle cerebral artery occlusion without craniectomy in rats. Stroke 20: 84-91, 1989.

18. Kostandy BB: The role of glutamate in neuronal ischemic injury: the role of spark in fire. Neurol Sci 33: 223-237, 2012.

19. Szydlowska K and Tymianski M: Calcium, ischemia and excitotoxicity. Cell Calcium 47: 122-129, 2010.

20. Henson MA, Roberts AC, Pérez-Otaño I and Philpot BD: Influence of the NR3A subunit on NMDA receptor functions. Prog Neurobiol 91: 23-37, 2010.

21. Shen H, Chen GJ, Harvey BK, Bickford PC and Wang Y: Inosine reduces ischemic brain injury in rats. Stroke 36: 654-659, 2005.

22. Shen H, Kuo CC, Chou J, et al: Astaxanthin reduces ischemic brain injury in adult rats. FASEB J 23: 1958-1968, 2009.

23. Hardingham GE, Fukunaga Y and Bading H: Extrasynaptic NMDARs oppose synaptic NMDARs by triggering CREB shut-off and cell death pathways. Nat Neurosci 5: 405-414, 2002.

24. Hoyte L, Barber PA, Buchan AM and Hill MD: The rise and fall of NMDA antagonists for ischemic stroke. Curr Mol Med 4 131-136, 2004

25. Liu Y, Wong TP, Aarts M, et al: NMDA receptor subunits have differential roles in mediating excitotoxic neuronal death both in vitro and in vivo. J Neurosci 27: 2846-2857, 2007.

26. Terasaki Y, Sasaki T, Yagita Y, et al: Activation of NR2A receptors induces ischemic tolerance through CREB signaling. J Cereb Blood Flow Metab 30: 1441-1449, 2010.
27. Cavara NA and Hollmann M: Shuffling the deck anew: how NR3 tweaks NMDA receptor function. Mol Neurobiol 38: 16-26, 2008.

28. Stys PK and Lipton SA: White matter NMDA receptors: an unexpected new therapeutic target? Trends Pharmacol Sci 28: 561-566, 2007.

29. Piña-Crespo JC, Talantova M, Micu I, et al: Excitatory glycine responses of CNS myelin mediated by NR1/NR3 'NMDA' receptor subunits. J Neurosci 30: 11501-11505, 2010.

30. Chatterton JE, Awobuluyi M, Premkumar LS, et al: Excitatory glycine receptors containing the NR3 family of NMDA receptor subunits. Nature 415: 793-798, 2002.

31. Fukumori R, Takarada T, Nakamichi N, et al: Requirement of both NR3A and NR3B subunits for dominant negative properties on $\mathrm{Ca}^{2+}$ mobilization mediated by acquired $\mathrm{N}$-methyl-D-aspartate receptor channels into mitochondria. Neurochem Int 57: 730-737, 2010.

32. Nakanishi N, Tu S, Shin Y, et al: Neuroprotection by the NR3A subunit of the NMDA receptor. J Neurosci 29: 5260-5265, 2009.

33. Zhu DY, Lau L, Liu SH, Wei JS and Lu YM: Activation of cAMP-response-element-binding protein (CREB) after focal cerebral ischemia stimulates neurogenesis in the adult dentate gyrus. Proc Natl Acad Sci USA 101: 9453-9457, 2004

34. Walton MR and Dragunow I: Is CREB a key to neuronal survival? Trends Neurosci 23: 48-53, 2000.

35. Finkbeiner S: CREB couples neurotrophin signals to survival messages. Neuron 25: 11-14, 2000. 